\title{
Regulatory Non-coding RNAs for Death Associated Protein Kinase Family
}

\author{
Qingshui Wang ${ }^{1,2 \dagger}$, Youyu Lin $^{2 \dagger}$, Wenting Zhong ${ }^{2 \dagger}$, Yu Jiang ${ }^{3 *}$ and Yao Lin ${ }^{1,2 *}$ \\ ${ }^{1}$ Central Laboratory at the Second Affiliated Hospital of Fujian Traditional Chinese Medical University, Collaborative Innovation \\ Center for Rehabilitation Technology, Fujian University of Traditional Chinese Medicine, Fuzhou, China, ${ }^{2}$ Key Laboratory of \\ Optoelectronic Science and Technology for Medicine of Ministry of Education, College of Life Sciences, Fujian Normal University, \\ Fuzhou, China, ${ }^{3}$ Prenatal Diagnosis Centre, Women and Children's Hospital, School of Medicine, Xiamen University, Xiamen, \\ China
}

The death associated protein kinases (DAPKs) are a family of calcium dependent serine/ threonine kinases initially identified in the regulation of apoptosis. Previous studies showed that DAPK family members, including DAPK1, DAPK2 and DAPK3 play a crucial regulatory role in malignant tumor development, in terms of cell apoptosis, proliferation, invasion and metastasis. Accumulating evidence has demonstrated that non-coding RNAs, including microRNA (miRNA), long non-coding RNA (IncRNA) and circRNA, are involved in the regulation of gene expression and tumorigenesis. Recent studies indicated that noncoding RNAs participate in the regulation of DAPKs. In this review, we summarized the current knowledge of non-coding RNAs, as well as the potential miRNAs, IncRNAs and circRNAs, that are involved in the regulation of DAPKs.

Keywords: DAPK, non-coding RNA, miRNA, IncRNA, circRNA

\section{DEATH-ASSOCIATED PROTEIN KINASE FAMILY}

DAPK is a family of serine/threonine kinases that belong to the calmodulin regulated kinase super family (Huang et al., 2014). At present, the most studied DAPK family member is DAPK1 (Lin et al., 2009; Stevens et al., 2009; Chen et al., 2014; Fujita and Yamashita, 2014; Yung et al., 2019). DAPK1 was identified in an unbiased antisense based genetic screen for genes whose protein products were necessary for interferon gamma (IFN- $\gamma$ ) induced death in HeLa cells (Lai and Chen, 2014; Liu et al., 2016). DAPK1 has a unique multi-domain structure, and the sequence from the $\mathrm{N}$ end to the $\mathrm{C}$ end is: a kinase domain, a calmodulin regulatory domain, eight continuous ankyrin repeats, two potential P-loop binding regions of Ras of Complex proteins (C-terminal of ROC), a death domain and a serine-rich tail. DAPK2 is comprised of a kinase domain, a calmodulin-binding autoregulatory domain and a C-terminal tail. DAPK3 contains kinase domain, a leucine zipper domain, and two putative nuclear localization sequences (NLS) (Shiloh et al., 2014) (Figure 1).

DAPK1 is involved in the regulation of cell apoptosis, autophagy and migration (Lin et al., 2007; Harrison et al., 2008; Gandesiri et al., 2012; Rennier and Ji, 2013a; Rennier and Ji, 2013b; Benderska et al., 2014; Rennier and Ji, 2015; Tian et al., 2016). Previous research revealed that DAPK1 is a tumor suppressor gene, and the level of DAPK1 was down regulated in various cancers (Raval et al., 2007; Kilinc et al., 2012; Gao et al., 2015; Cai et al., 2017; Xie et al., 2017; Steinmann et al., 2019). In the early stage of cancer, DAPK1 can suppress tumor growth and metastasis by increasing apoptosis. In late cancer, DAPK1 can inhibit cell movement and adhesion by interfering with integrins (Kuo et al., 2006). In contrast to DAPK1, DAPK2 has not been identified as a tumor suppressor in solid tumors. 


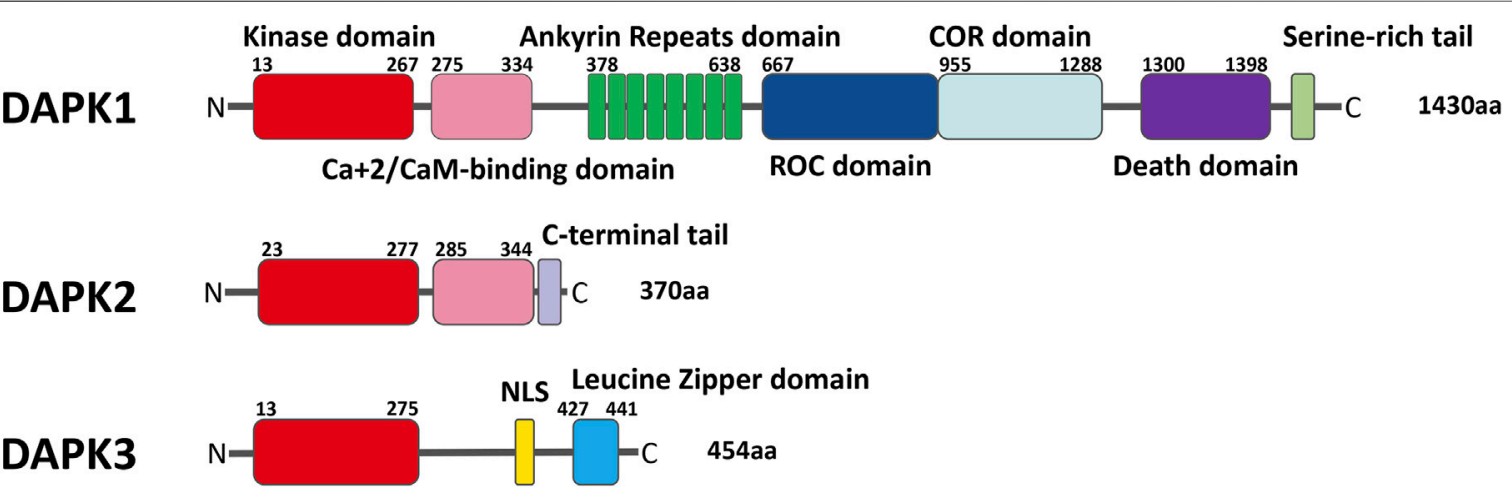

FIGURE 1 | Schematic representation of the domains of each of the DAPK family members with their respective location.

DAPK2 is mainly expressed in the hematopoietic compartment. At present, DAPK2 has been found to act as a tumor suppressor in several types of leukemia (Rizzi et al., 2007; Humbert et al., 2014; Ye et al., 2016). DAPK3 is considered as a tumor suppressor. Previous reports revealed that the DAPK3 promoter is often methylated in various types of cancer, resulting in the loss of its tumor suppressor effect (Brognard et al., 2011; Das et al., 2016).

\section{METHYLATION OF DEATH-ASSOCIATED PROTEIN KINASE GENE IN TUMOR}

Hypermethylation of DAPK has been frequently described in many different cancers compared with normal tissues, including esophageal, breast malignancies, head and neck, kidney and bladder, ovary, B cell lymphoma and lung cancer (SanchezCespedes et al., 2000; Lehmann et al., 2002; Brock et al., 2003; Christoph et al., 2006; Collins et al., 2006). Raveh et al. found that the methylation of DAPK1 gene accounts for about $20 \%$ in primary colon cancer patients. Bing et al. found that the methylation frequency of DAPK1 gene was approximately 17.7 and $54.8 \%$ in normal gastric tissue and gastric cancer tissue samples respectively (Ye et al., 2012). Harder et al. revealed that the methylation of DAPK1 gene accounted for about $68 \%$ in 34 liver cancer samples, but in 16 normal liver tissues, its methylation accounted for about 31\% (Harder et al., 2008). In addition, Flatley et al. revealed that cervical cancer cells whose DAPK1 gene is methylated may be more susceptible to human papillomavirus (HPV) infection (Flatley et al., 2014).

Abnormal promoter methylation is an early and frequent event in the process of cell carcinogenesis, and can be used as a sensitive biomarker of tumorigenesis. Bing et al. pointed out that the methylation frequency of the DAPK1 promoter in gastric cancer tissue samples was three times higher than that in normal gastric tissue. That is, along with the process of gastric carcinogenesis, the methylation rate of DAPK1 gene will increase, so it can be speculated that DAPK1 can be used as a marker of gastric cancer (Ye et al., 2012). Research by Krajnovic et al. pointed out that DAPK1 gene methylation accounted for $79 \%$, and O-6 methylguanine DNA methyltransferase (MGMT) gene methylation accounted for 59\% in 32 follicular lymphoma (FL) samples. And the synergistic methylation of these two genes can be used as a marker of FL disease recurrence and drug resistance (Krajnović et al., 2013).

Although there have many relevant reports on the detection of the methylation frequency of the DAPK1 promoter in multiple patient samples. However, due to the limited patient information and the number of samples. There are few studies on the analysis of the methylation of DAPK1 gene, the mRNA level of DAPK1, and the protein level of DAPK1 in the same patient sample. It is necessary to simultaneously detect these three levels in appropriate samples to further understand the biological function and clinical significance of DAPK1 gene methylation in tumors. Our previous study found that DNA methylation status of DAPK1 did not correlate well with its mRNA or protein expression in breast cancer, which indicated that DAPK1 expression is not only regulated by methylation (Zhu et al., 2017). In addition to the methylation, there has been an increasing number of studies on the association between regulation of DAPKs and non-coding RNAs.

\section{NON-CODING RNAS}

In recent years, non-coding RNA (ncRNA) has received increasing attention (Guo et al., 2015; Esposito et al., 2016; Sun et al., 2018; Fridrichova and Zmetakova, 2019; Ban et al., 2020; Zhang et al., 2020). With more and more non-coding RNAs being identified, their functions have been gradually discovered. In 1993, Ambros and Ruvkun announced the discovery of miRNA which is related to the development and regulation of Caenorhabditis elegans. After that, hundreds of similar short RNAs were found in drosophila, human and worm cells (Cech and Steitz, 2014). Non-coding RNAs regulate target genes at transcription level and RNA processing level, and participate in almost all physiological processes such as embryo development, protein synthesis, cell differentiation and 


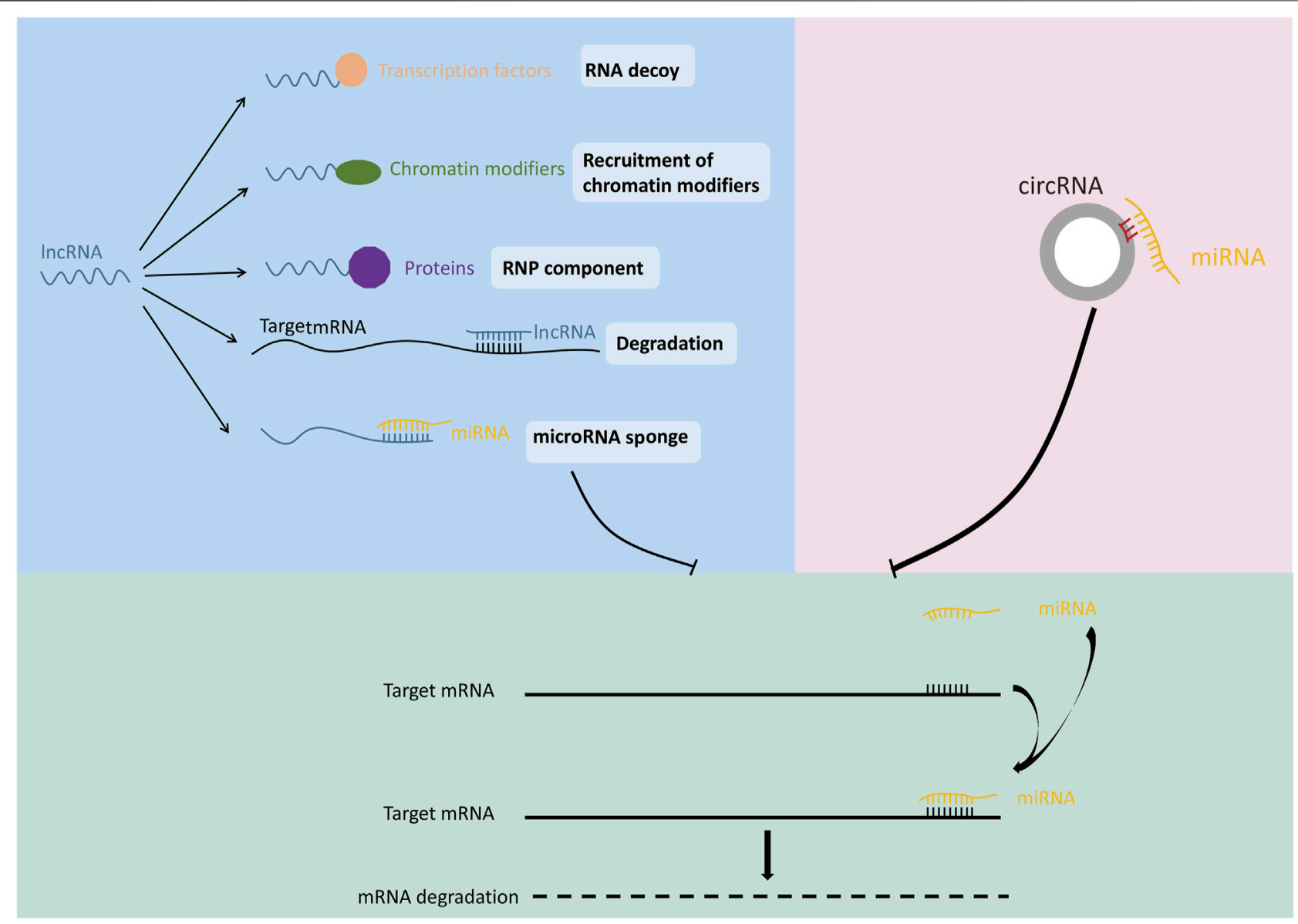

FIGURE 2 | Mechanisms of ncRNA function.

apoptosis (Cech and Steitz, 2014). There are two major types of non-coding RNA with regulatory functions according to their size: short chain non-coding RNAs (siRNA, miRNA and piRNA) with length between several and $200 \mathrm{nt}$, and long chain non-coding RNAs (lncRNA) with length greater than $200 \mathrm{nt}$. In recent years, a number of non-coding circular RNAs (circRNAs) have also been identified (Hsiao et al., 2017). Currently, miRNA, lncRNA and circRNA are the most studied non-coding RNAs.

MiRNA is a family of highly conserved, non-coding RNAs encoding 19-25 nt. The main function of miRNA is its participation in the regulation of gene expression after transcription (Wang et al., 2018a). MiRNA can play a suppressive role by binding to the mRNA of target gene sequence often locating on the $3^{\prime} \mathrm{UTR}$, thereby inhibiting target gene expression (Figure 2) (Bushati and Cohen, 2007; Chen et al., 2019; Michlewski and Cáceres, 2019; Sur et al., 2019). Dysregulation of miRNA has been confirmed to be closely related to cancer and many other diseases (Liu and Li, 2019; Zhao et al., 2019). Previous studies revealed that the expression patterns were different for each miRNA among different cancer tissues. For example, miR-34 was down-regulated in prostate cancer, breast cancer, lung cancer and osteosarcoma compared with normal tissues, but was up-regulated in liver cancer (Zhang et al., 2019a). These studies suggested that same gene may have a completely different expression pattern in different types of tumors due to the regulation of miRNA.

LncRNA refers to non-coding RNA transcripts with length greater than $200 \mathrm{nt}$ (Jathar et al., 2017). With the continuous progress of genomics and exploration of lncRNA family members, the regulatory effect of $\operatorname{lncRNA}$ on gene function has been gradually discovered (Chen, 2016). LncRNAs exert functions at different positions according to different mechanisms such as regulation of transcription, translation, protein modification and the formation of RNA-protein or protein-protein complexes (Figure 2) (Xing et al., 2015; Botti et al., 2017; Renganathan and Felley-Bosco, 2017; Wang et al., 2018b; Liao et al., 2018). The competing endogenous RNA (ceRNA) is the most studied mechanism of lncRNA-mediated gene expression regulation. LncRNA can act as a miRNA sponge via ceRNA mechanism, thereby regulating the expression of miRNA target genes (Iwakiri et al., 2017; Militello et al., 2017; Chen et al., 2018a). For example, lncRNA ATB was deregulated in hepatocellular carcinoma, lung cancer and other malignant tumors, and caused tumor occurrence and development through ceRNA mechanism ( $\mathrm{Li}$ et al., 2017a). LncRNA DANCR was deregulated in lung cancer, and promoted tumor 
growth and invasion by binding with tumor suppressor miR-216a (Zhen et al., 2018).

CircRNA is a new type of non-coding RNA with covalently closed ring structure. The formation of circRNA is through the reverse splicing process in the splicing modification of precursor mRNA, which makes the splicing donor at the 3'end of pre-mRNA connect with the splicing receptor at the $5^{\prime}$ end (Chen and Yang, 2015). Since circRNA involves a wide range of biological processes, the deregulation of circRNA may lead to abnormal cell function and diseases (Zhang et al., 2018a; Bi et al., 2018; Chen et al., 2018b; Lin and Chen, 2018; Zhang et al., 2019b). For example, the expression of circSLC8A1 was decreased in bladder cancer. Overexpression of circSLC8A1 inhibited bladder cancer cell proliferation, migration and invasion. Further study revealed that circSLC8A1 acts as a miRNA sponge for miR-494 and miR-130b, and subsequently regulate the expression of their target gene PTEN (gene of phosphate and tension homology deleted on chromsome ten), thereby suppressed the progression of bladder cancer (Lu et al., 2019). Similarly, a large amount of circRNAs have been identified to enhance the transcription of target genes through ceRNA mechanisms (Figure 2) (Zhang et al., 2018b; Fang et al., 2018; Zhu et al., 2018; Cao et al., 2019; Costa et al., 2019; Liu et al., 2019; Yu and Liu, 2019; Yuan et al., 2019; Chen et al., 2020).

\section{THE REGULATORY NON-CODING RNAS FOR DEATH-ASSOCIATED PROTEIN KINASES}

\section{Non-coding RNAs Involved in the Transcription Regulation of Death-Associated Protein kinase1}

Emerging evidence showed that numerous miRNAs caused DAPK1 deregulation in multiple cancers, for example, miR103, miR-34a-5p and miR-191 etc.

In colorectal cancer, Chen et al. found that the increased levels of miR-103 and miR-107 were related to the metastasis potential of tumor cells by targeting DAPK1 and KLF4 (Kruppel like factor 4). In renal cell carcinoma (RCC), the expression of DAPK1 was decreased. Functionally, DAPK1 overexpression repressed RCC cell proliferation, migration and invasion. Meanwhile, the expression of DAPK1 was identified to be regulated by miR-34a (Jing et al., 2019). In ovarian cancer, knockdown of DAPK1 could weaken its response to TNF-ainduced cell death in CRL-7566 cells. Higher level of miR-191 in ovarian cancer patient samples compared with controls was verified, and miR-191 was confirmed to directly target DAPK1 and regulate its expression using luciferase assay. Further, the authors demonstrated that the miR-191-DAPK1 axis plays a major role in modulating the response of endometrioid carcinoma cells to death-inducers (Tian et al., 2015; Vastrad et al., 2017). In gliomas and glioblastoma, miR-103a-3p-DAPK1 axis and miR-22-3p-DAPK1 axis might be associated with the diagnosis and treatment of gliomas and glioblastoma (Vastrad et al., 2017). In nasopharyngeal carcinoma (NPC), the over-expression of miR483-5p decreased the radiosensitivity of NPC cells in vivo and in vitro. MiR-483-5p decreased radiation-induced apoptosis and DNA damage, and increased NPC cell colony formation by targeting DAPK1 (Tian et al., 2019). These results implied that modulation of miR-483-5p-DAPK1 axis may provide a new approach for increasing the radiosensitivity of NPC. In pancreatic cancer, the level of DAPK1 was significantly downregulated, and increased DAPK1 expression inhibited the migration and invasion of pancreatic cancer cells. MiR-182 was highly expressed in pancreatic cancer and confirmed to directly target DAPK1. MiR-182-DAPK1 axis has been demonstrated to plays an important role in the development and progression of pancreatic cancer (Xu et al., 2017). In endometrial cancer (EC), lncRNA MIR22 was reported to be significantly down-regulated in endometrial cancer tissues. Functionally, over-expression of lncRNA MIR22 significantly inhibited endometrial cancer cells proliferation, induced EC cells apoptosis, and arrested endometrial cancer cells in G0/G1 phase. Furthermore, miR-141-3p was identified as a target for lncRNA MIR22. Subsequently, lncRNA MIR22 was found to inhibit endometrial cancer cell proliferation by regulating the miR-141-3p-DAPK1 axis (Cui et al., 2018). In breast cancer, hypermethylation of miR-127 was observed in 58 breast cancer tissues compared with paired normal breast tissues. Clinical research data indicated that the up-regulated expression levels of DAPK1 in breast cancer patients were negatively correlated with the decreased expression of miR-127-5p. In addition, the miR-127DAPK1 axis was found to be related to breast cancer progression, particularly metastasis (Pronina et al., 2017).

In addition to cancer, the expression of DAPK1 was also regulated by non-coding RNAs in numerous other diseases. In rheumatoid arthritis (RA), miR-103a-3p was significantly upregulated in the whole blood and peripheral blood mononuclear cells of RA patients compared with healthy control (Anaparti et al., 2017). Additionally, DAPK1 was found to be a target of miR-103a3pand decreased in RA. In Parkinson's disease (PD), the level of DAPK1 was increased in PD mice and positively correlated with synucleinopathy, and DAPK1 is a target of miR-26. MiR-26 knockdown or over-expression of DAPK1 resulted in synucleinopathy, dopaminergic neuron cell death, and motor disabilities in wild-type mice. Further investigation revealed that miR-26-DAPK1 axis were essential in the formation of the molecular and cellular pathologies in PD (Su et al., 2019). In cardiac ischemia-reperfusion (IR) injury, the level of miR-98 was decreased in the cardiomyocytes subjected to hypoxia/reoxygenation $(\mathrm{H} / \mathrm{R})$ and in the myocardium of the $\mathrm{I} / \mathrm{R}$ rats. Moreover, increased miR-98 expression could significantly reduce the myocardial oxidative stress, ischemic injury and cell apoptosis. Subsequently, DAPK1was confirmed as a direct target of miR-98 using luciferase activity assay (Zhai et al., 2019). In chronic obstructive pulmonary disease (COPD), PM2.5 significantly aggravated apoptosis in cigarette-inflamed Human bronchial epithelial cells (HBEpiCs). The level of miR-194-3p was detected to be down-regulated in PM2.5-CSS-treated HBEpiCs. Bioinformatics and luciferase activity assay reported that DAPK1 was directly targeted by miR-194-3p. Inhibition of miR-194-3p increased DAPK1 expression and apoptosis in normal HBEpiCs (Zhou et al., 2018). In ischemic stroke, increased lncRNA AK038897 and decreased miR-26a-5p levels were observed in mouse brains following middle cerebral 
TABLE 1 | Non-coding RNAsin the regulation of DAPKs.

\begin{tabular}{|c|c|c|c|}
\hline Number & Cancer or Disease & Target gene & Non-coding RNAs \\
\hline 1 & Colorectal cancer & DAPK1 & miR-103 \\
\hline 2 & Renal cell carcinoma & DAPK1 & miR-34a-5p \\
\hline 3 & Ovarian cancer & DAPK1 & miR-191 \\
\hline 4 & Breast cancer & DAPK1 & $\operatorname{miR}-127-5 p$ \\
\hline 5 & Gliomas and glioblastoma & DAPK1 & miR-103a-3p, miR-22-3p \\
\hline 6 & Nasopharyngeal carcinoma & DAPK1 & $\operatorname{miR}-483-5 p$ \\
\hline 7 & Endometrial cancer & DAPK1 & MIR22HG/miR-141-3p \\
\hline 8 & Rheumatoid arthritis & DAPK1 & $\operatorname{miR}-103 a-3 p$ \\
\hline 9 & Parkinson's disease & DAPK1 & $\operatorname{miR}-26$ \\
\hline 10 & Cardiac ischemia-reperfusion & DAPK1 & $\operatorname{miR}-98$ \\
\hline 11 & Chronic obstructive pulmonary disease & DAPK1 & $\operatorname{miR}-194-3 p$ \\
\hline 12 & Ischemic stroke & DAPK1 & AK038897/miR-26a-5p \\
\hline 13 & Polycystic ovary syndrome & DAPK1 & $\operatorname{miR}-141-3 p$ \\
\hline 14 & Epithelial ovarian cancer & DAPK2 & miR-520g \\
\hline 15 & Breast cancer & DAPK2 & miR-520h \\
\hline 16 & Gastric cancer & DAPK2 & miR-34a, miR-135a \\
\hline 17 & Colorectal cancer & DAPK2 & $\operatorname{miR}-1285-3 p$ \\
\hline 18 & Diabetic cardiomyopathy & DAPK2 & MIAT/miR-22-3p \\
\hline 19 & Ischemia reperfusion injury & DAPK2 & miR-133a \\
\hline 20 & Breast cancer & DAPK3 & miR-17/20a \\
\hline 21 & Ovarian cancer & DAPK3 & miR-1307 \\
\hline
\end{tabular}

artery occlusion/reperfusion (MCAO/R) and in neuro-2A (N2a) neuroblastoma cells following oxygen-glucose deprivation and reoxygenation (OGD/R). Further studies showed that AK038897 and DAPK1 were targets of miR-26a-5pusing luciferase activity assay. Finally, AK038897 -miR-26a-5p- DAPK1 axis was considered as a key mechanism controlling cerebral ischemia injury (Wei et al., 2019). In polycystic ovary syndrome (PCOS), the level of miR-141$3 p$ was decreased in the ovaries of rat PCOS models, and increased level of miR-141-3p decreased cells apoptotic rate (Li et al., 2017b). Luciferase activity assay reported that DAPK1 was the target of miR141-3p, indicating that miR-141-3p is involved in the etiology of PCOS by targeting DAPK1 (Table 1; Figure 3).

\section{Non-coding RNAs Involved in the Transcription Regulation of Death-Associated Protein kinase2}

The expression of DAPK2 was also reported to be regulated by noncoding RNAs in cancers and other diseases. In epithelial ovarian cancer (EOC), the level of miR-520g was significantly increased in EOC tissues compared with normal tissues, and over-expression of miR-520g increased EOC cell proliferation and promoted cell invasion (Zhang et al., 2016). DAPK2 was a target of miR-520g. MiR-520g knockdown or DAPK2 over-expression reduced EOC cell proliferation, invasion and chemoresistance. These results suggested that miR-520g promoted EOC progression and drug resistance by regulating DAPK2. In breast cancer, miR-520h could promote the drug resistance of breast cancer cells (Su et al., 2016). Bioinformatics prediction, compensatory mutation and functional validation further confirmed that DAPK2 is a target of miR-520h. Over-expression of DAPK2 could abolish breast cancer cell drug resistance induced by miR-520h, suggesting miR-520h-DAPK2 axis is a potential functional target for breast cancer therapy. In gastric cancer, the level of miR-34a was significantly increased and correlated with dendritic cell mediated enhancement of anti-tumor immunity against gastric cancer cell.
DAPK2 and Sp1 were targets of miR-34a (Yan et al., 2016a). Another research found that miR-135a was highly expressed in gastric cancer tissues compared to normal tissues. Gastric cancer with high miR135a level was more likely to display aggressive characteristics. Moreover, miR-135a promoted the proliferation and invasion of oxaliplatin-resistant gastric cancer cells, and inhibited E2F transcription factor 1 (E2F1) induced apoptosis by inhibiting E2F1 and DAPK2 expression (Yan et al., 2016b). In colorectal cancer, miR1285-3p improved colorectal cancer cell proliferation and escape from apoptosis by targeting DAPK2 (Villanova et al., 2020). In diabetic cardiomyopathy (DCM), IncRNA myocardial infarction associated transcript (MIAT) was significantly upregulated in the rat model of DCM. Knockdown of MIAT reduced cardiomyocyte apoptosis and improved left ventricular function in diabetic rats. Luciferase activity assay reported that MIAT and DAPK2 were directly targeted by miR-22-3p (Zhou et al., 2017). MIAT may function as a competing endogenous RNA to positively regulate DAPK2 expression by sponging miR-22-3p, which consequently leads to cardiomyocyte apoptosis involved in the pathogenesis of DCM. In ischemia reperfusion injury, DAPK2 was confirmed as a target of miR-133a. In H9C2 cells, ischemia reperfusion caused a sharp decrease in miR-133a expression and a significant upregulation of DAPK2 expression (Li et al., 2015) (Table 1; Figure 3).

\section{Non-coding RNAs Involved in the Transcription Regulation of Death-Associated Protein kinase 3}

Compared to DAPK1 and DAPK2, fewer studies investigated the association between DAPK3 and non-coding RNAs. DAPK3 was reported to be a target of miR-17/20a and played an important role in preventing miR-17/20a depletion-induced genome instability or miR-17/20a overexpression triggered tumor formation (Cai et al., 2015). In ovarian cancer, miR-1307 was up-regulated in the chemoresistant epithelial ovarian cancer tissues and might play 

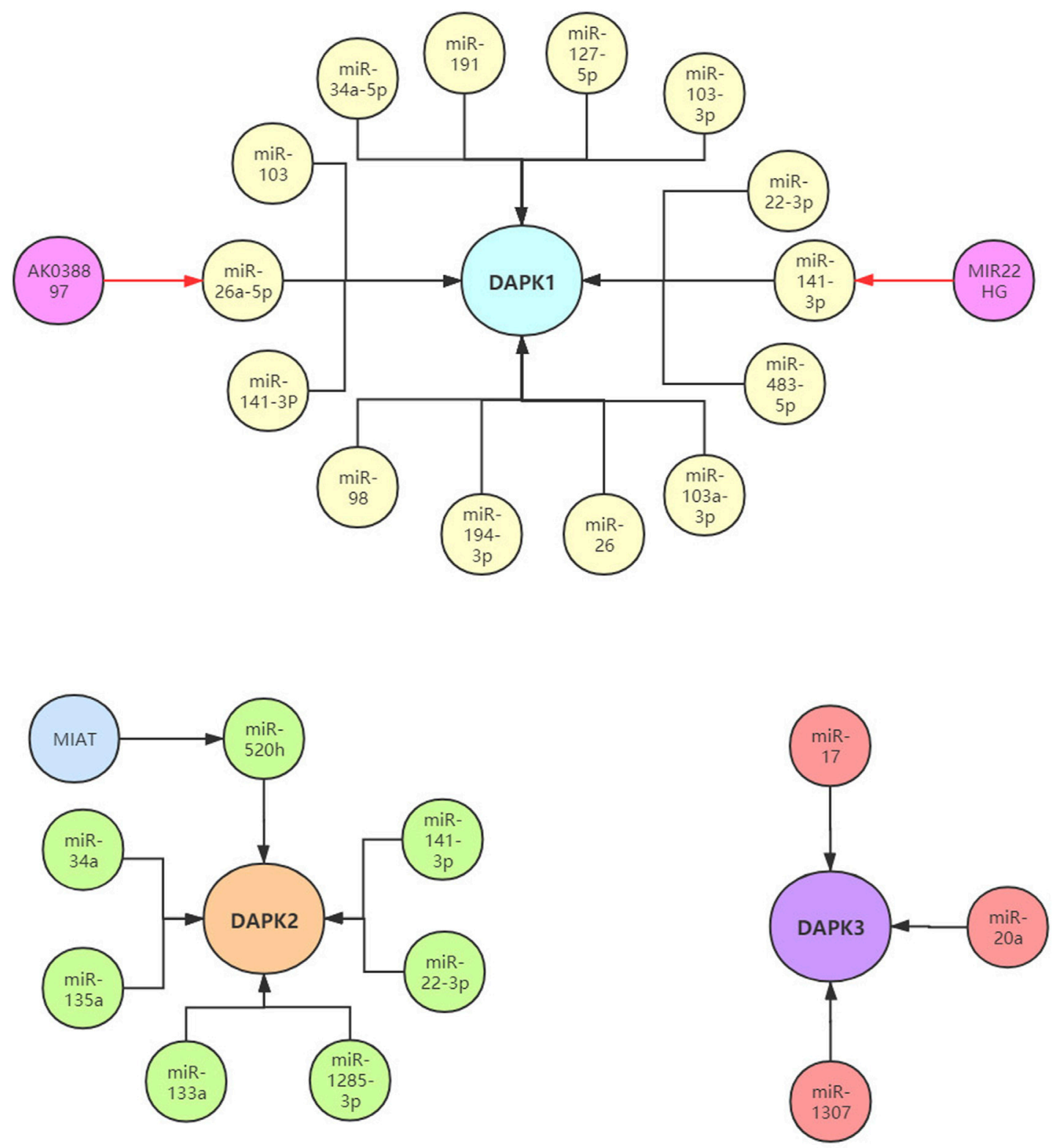

FIGURE 3 | The reported non-coding RNAs in the regulation of DAPKs.

a role in the development of chemoresistance in ovarian cancer by targeting DAPK3 (Zhou et al., 2015) (Table 1; Figure 3).

\section{BIOINFORMATICS ANALYSIS OF POTENTIAL NON-CODING RNAS INVOLVED IN THE TRANSCRIPTION REGULATION OF DEATH-ASSOCIATED PROTEIN KINASES}

Although there have been many studies on the non-coding RNAs regulating DAPKs, bioinformatic tools are available to computationally predict new non-coding RNAs for DAPKs, which may help us have a better understanding about the regulation of DAPKs.

\section{Analysis of Potential miRNAs Involved in the Transcription Regulation of Death-Associated Protein kinases}

By analyzing a large set of Ago and RBP (RNA binding protein) binding sites derived from all available CLIP-Seq experimental techniques (PAR-CLIP, HITS-CLIP, iCLIP, CLASH), ENCORI (http://starbase.sysu.edu.cn/index.php) have shown extensive and complex RNA-RNA interaction networks (Li et al., 2014; 
A

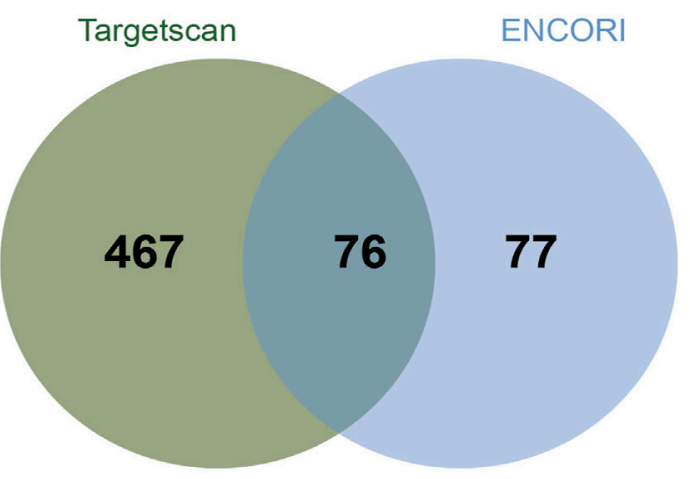

DAPK1

C Targetscan

ENCORI

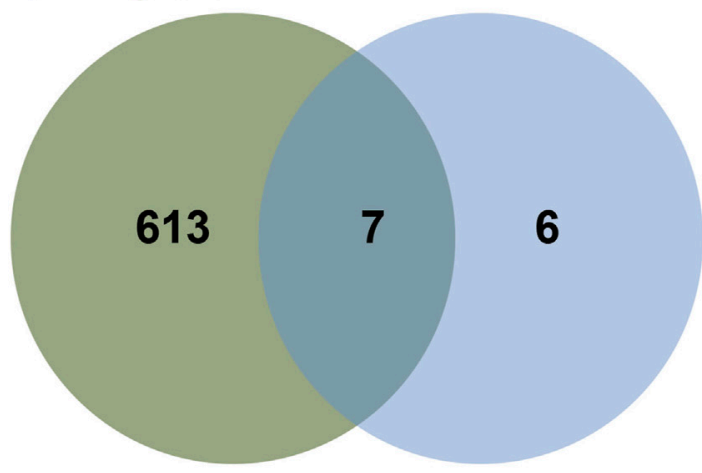

DAPK2

$\mathbf{E}$

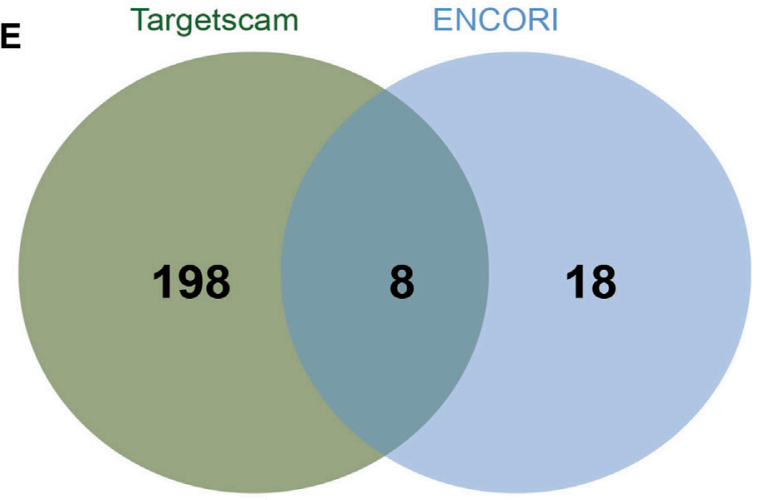

DAPK3

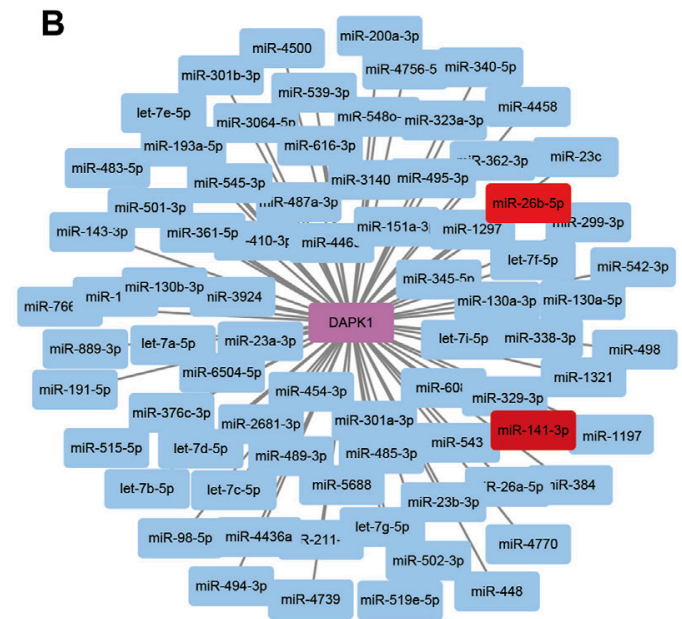

D

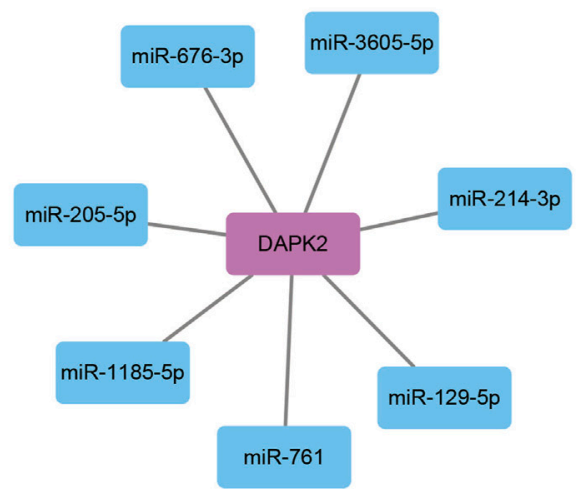

F

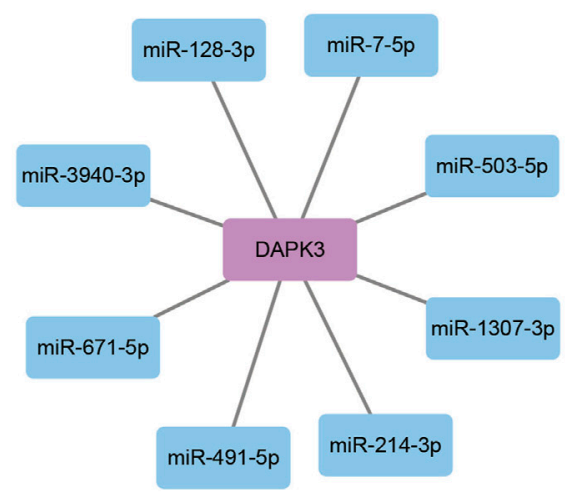

FIGURE 4 | Bioinformatics analysis of potential miRNAs in the regulation of DAPKs. Venn diagrams show potential miRNAs in the regulation of DAPK1 (A), DAPK2 (C) and DAPK3 (E) based on Targetscan and ENCORI website. Network connection diagram showing potential miRNAs in the regulation of DAPK1 (B), DAPK2 (D) and DAPK3 (F) based on Targetscan and ENCORI website. 


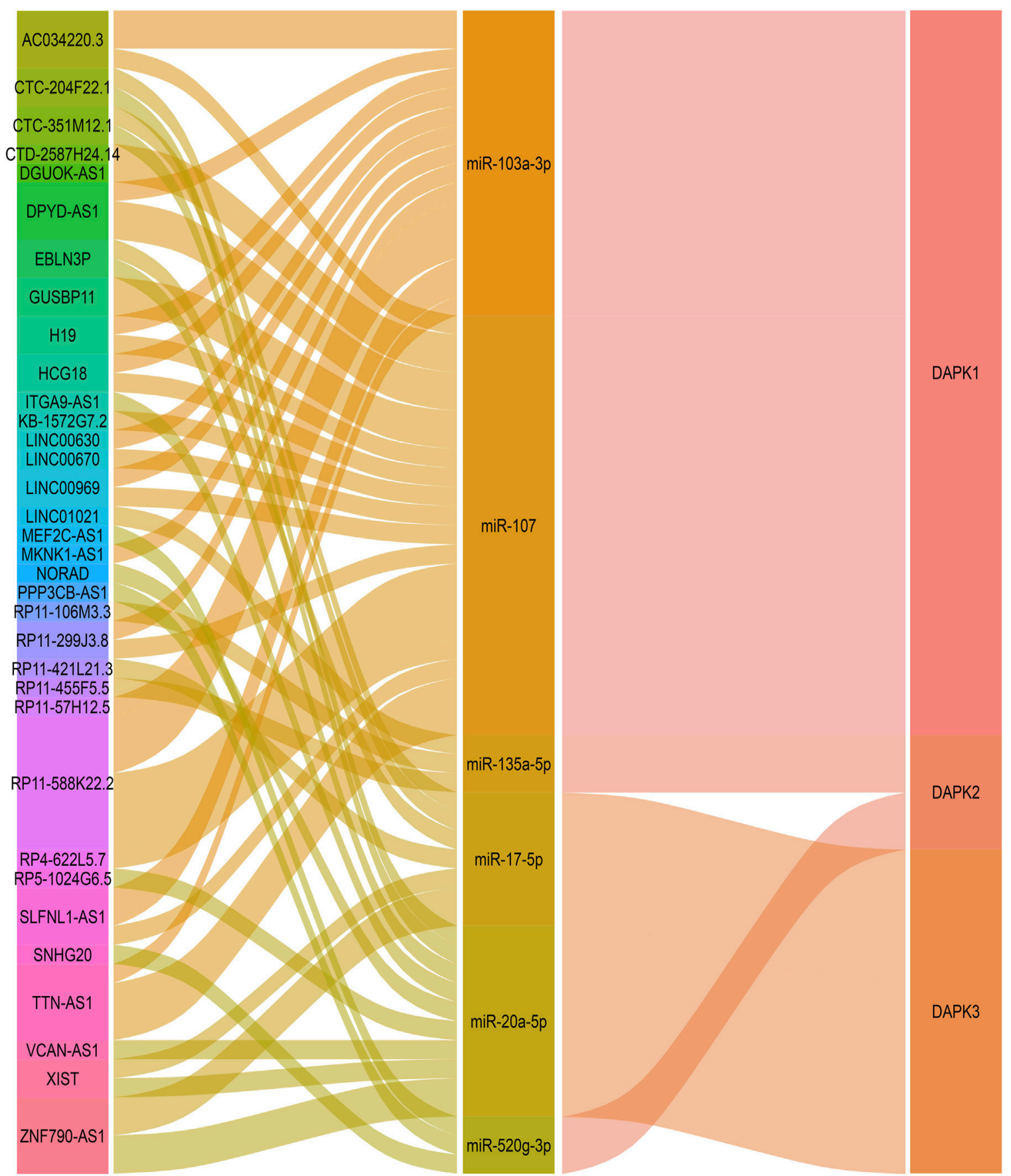

FIGURE 5 | Bioinformatics analysis of potential IncRNAs in the regulation of DAPKs. Sankey diagram for the ceRNA network in DAPK1. Each rectangle represents a gene, and the connection degree of each gene is visualized based on the size of the rectangle.

Fan et al., 2020). Targetscan (http://www.targetscan.org/vert_72/ ) predicts biological targets of miRNAs by searching for the presence of conserved sites that match the seed region of each miRNA (Agarwal et al., 2015). The overlapping target genes in these two databases were considered as miRNA-target genes. According to the prediction of Targetscan and ENCORI databases, 543 miRNAs and 153 miRNAs for DAPK1 were identified respectively. The intersection of the two predictions includes 76 miRNAs such as miR-362-3p, miR-329-3p, miR-1413p, miR-26b-5p, miR-483-5p and miR-98-5p et al. (Figures 4A,B). Among them, miR-141-3p and miR-26b-5p have been reported to regulate DAPK1 expression (Cui et al., 2018; Su et al., 


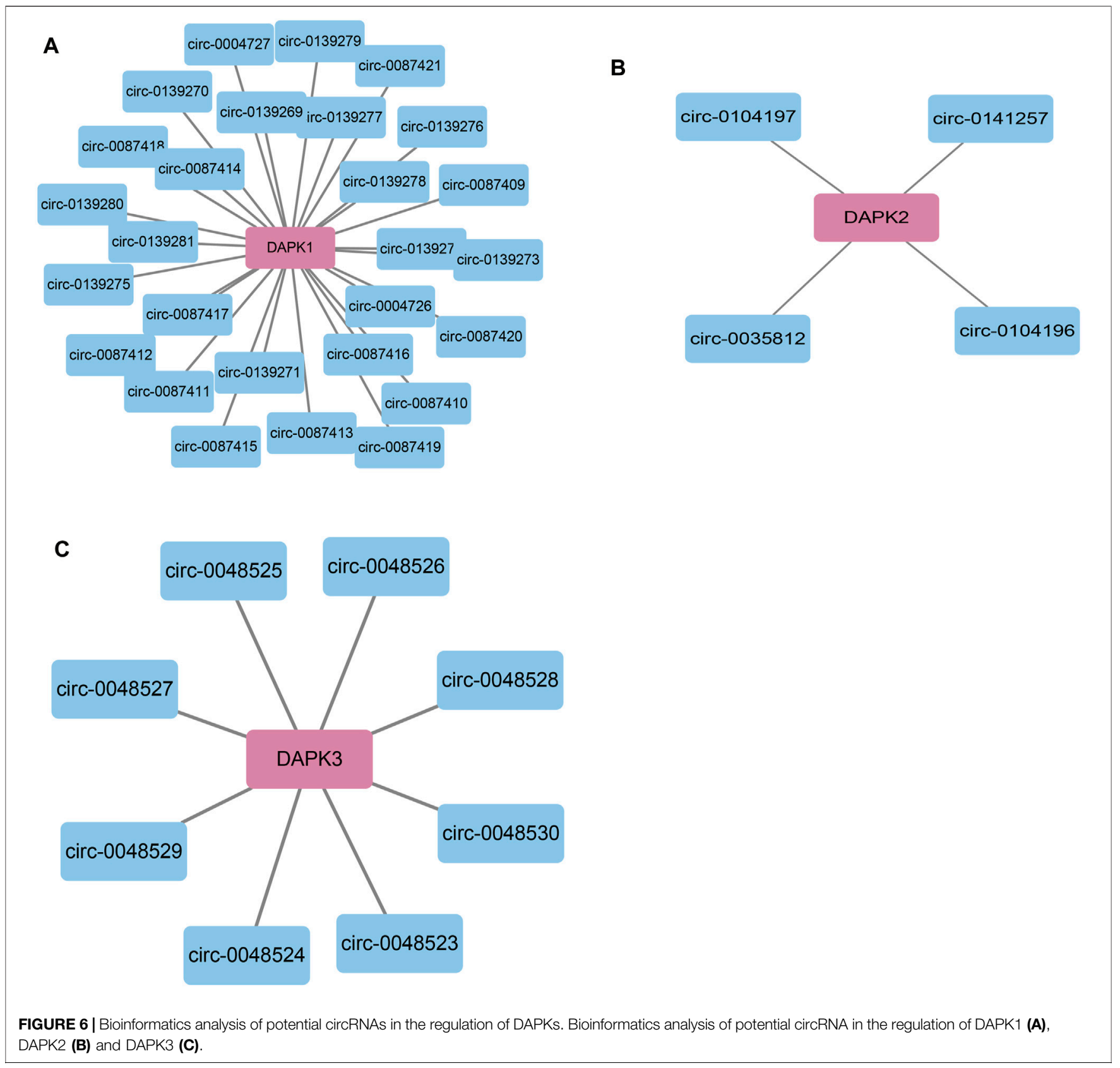

2019). Similarly, the intersection for DAPK2 and DAPK3 were seven miRNAs and eight miRNAs respectively (Figures 4C-F).

\section{Analysis of Potential IncRNAs Involved in the Transcription Regulation of Death-Associated Protein kinases}

LncACTdb 2.0 (http://www.bio-bigdata.net/LncACTdb/) is a database which provides comprehensive information of competing endogenous RNAs (ceRNAs) (Wang et al., 2019). We used LncACTdb 2.0 database to discover lncRNAs that may regulate DAPKs via ceRNAs mechanism. Base on the bioinformatics analysis, 38 lncRNA-miRNA-DAPK1 mRNA
ceRNA network, six lncRNA-miRNA-DAPK2 mRNA ceRNA network and 17 lncRNA-miRNA-DAPK3 mRNA ceRNA network were constructed (Figure 5). 38 lncRNA-miRNADAPK1 mRNA ceRNA network has 18 lncRNA including TTN-AS1, RP11-299J3.8, LINC00630, MKNK1-AS1, SLFNL1AS1, HCG18, RP11-57H12.5, CTD-2587H24.14, DGUOK-AS1, RP11-588K22.2, RP4-622L5.7, H19, GUSBP11, LINC00969, AC034220.3, DPYD-AS1, LINC00670 and KB-1572G7.2. GUSBP11 is significantly higher in gastric cancer plasma compared with healthy controls (Zheng et al., 2019). DGUOKAS1 promotes the proliferation cervical cancer by sponging miR653-5p and regulating EMSY (Yan et al., 2020). LINC00630 promotes radio-resistance by regulating BEX1 gene in 
colorectal cancer cells (Liu et al., 2020). HCG18 contributes to the progression of hepatocellular carcinoma via miR-214-3p/ CENPM (Zou et al., 2020). TTN-AS1 promotes the progression of lung adenocarcinoma, cholangiocarcinoma and colorectal cancer (Jia et al., 2019; Zhu et al., 2020a). H19 is abnormally expressed in human malignant tumors, and regulates cell proliferation, migration and via various mechanisms (Ye et al. , 2019). Six lncRNA-miRNA-DAPK2 mRNA ceRNA network has six lncRNA including PPP3CB-AS1, SNHG20, MEF2C-AS1, RP11-455F5.5, RP11-106M3.3 and LINC01021. It has been widely reported that SNHG20 is elevated in various cancers, indicating that SNHG20 may participate in cancer initiation and development (Zhu et al., 2020b). MEF2C-AS1 is identified as a novel biomarker in diffuse gastric cancer (Luo et al., 2018). 17 lncRNA-miRNA-DAPK3 mRNA ceRNA network has 10 lncRNA including ZNF790-AS1, NORAD, EBLN3P, XIST, RP5-1024G6.5, CTC-351M12.1, CTC-204F22.1, ITGA9-AS1, RP11-421L21.3 and VCAN-AS1. EBLN3P promotes the progression of liver cancer by regulating miR-144-3p/DOCK4 (Li et al., 2020). VCAN-AS1 contributes to the progression of gastric cancer via regulating p53 expression (Feng et al., 2020). NORAD is a highly conserved lncRNA necessary for genome stability and is dysregulated in various cancers (Soghli et al., 2021). XIST is associated with poor prognosis and metastasis of cancer in patients (Yin et al., 2019).

\section{Analysis of Potential circRNA Involved in the Transcription Regulation of Death-Associated Protein kinases}

CircBase (http://www.circbase.org/) is a database for circular RNAs (Glažar et al., 2014). Using CircBase, we found 27 circRNAs that may regulate DAPK1 including circ-0087415, circ-0139281 and circ-008742 et al., four circRNAs for DAPK2 including circ-0104197, circ-0141257, circ0104196 and circ-0035812 and eight circRNAs for DAPK3 including circ-0048524, circ-0048528, circ-0048527, circ0048525, circ-0048526, circ-0048530, circ-0048523 and circ-0048529 (Figure 6).

\section{CONCLUSION}

In the review, we conducted a comprehensive review of studies on non-coding RNAs and DAPKs to understand the current research status, and to explore the potential regulatory miRNAs, IncRNAs and circRNAs for the expression of DAPKs. Based on the review of current evidence, we have presented the regulatory network linking miRNAs, lncRNAs and DAPKs. It should be noted that much of our current understanding of non-coding RNAs in the transcription regulation of DAPKs comes from studies of a small number of non-coding RNAs and it is presently unclear whether these are representative of the group as a whole. Any review on this subject of non-coding RNAs in the regulation of DAPKs will always be incomplete for the reason that the field is still expanding. In addition to the miRNAs and IncRNAs described here, the
circRNAs participate in the regulation of DAPKs remains an unexplored area of investigation. One thing that seems likely is that as we begin to identify more non-coding RNAs regulating DAPKs, the regulatory mechanisms of DAPKs in cancers and disease will be better understood.

It is well known that one non-coding RNA regulates multiple genes, and one gene may be regulated by multiple non-coding RNAs. As demonstrated in this review, the expression of DAPK1 is regulated by multiple non-coding RNAs. One important line for future research will be to identify the key non-coding RNAs participating in the transcription regulation of DAPKs in specific types of cancer.

Bioinformatics analyses have in the past displayed a strong ability to predict and construct the coding gene and non-coding gene co-expression network. Previous study has in the past tended to focus only on the miRNAs in the transcription regulation of DAPKs. For example, DAPK1 has been confirmed to be regulated by miR-191, miR-483-5p, miR-141$3 p$, miR-98, miR-141-3p and miR-26a-5p. In prediction websites ENCORI and Targetscan, these miRNAs were predicted to bind to DAPK1 3' UTR. MiR-129-5p, miR-205-5p, miR-214-3p, miR1185-5p, miR-761, miR-3605-5p and miR-676-3p were found to be involved in the transcription regulation of DAPK2, these miRNAs were predicted to bind to DAPK2 $3^{\prime}$ UTR in Targetscan websites. Compared to miRNAs, there has been little research on lncRNAs and circRNAs targeting DAPKs, and only three lncRNAs were reported to be involved in the transcription regulation of DAPKs. As it becomes clear that lncRNA and circRNA have a distinct biological role, it is logical to discovered that lncRNA and circRNA that regulate DAPKs will be found to have clinical implications and also be worthy of investigation. Base on LncACTdb website, we found that a total of two miRNAs, miR-103a-3p and miR-107, were involved in the 38 lncRNA-miRNA-DAPK1 ceRNA network. Meanwhile, miR-103a-3p and miR-107 were also predicted to bind to DAPK1 3' UTR through ENCORI website. These results imply that miR-103a-3p and miR-107 may play an important role in the transcription regulation of DAPK1. Of additional concern, lncRNA DAPK1-IT1 is transcribed from an intron of the DAPK1 gene. DAPK1-IT1 has been reported to regulate cholesterol metabolism and inflammatory response in macrophages and promotes atherogenesis by sponging miR-590-3p and regulating LPL (lipoprotein lipase), and possibly linked to respiratory diseases. Thus, the interaction between DAPKs and the ncRNAs can be complex (Zhen et al., 2019; Liao et al., 2020).

In conclusion, this review integrates and predicts the potential non-coding RNAs that may participate in the transcription regulation of DAPKs. We hope this could help speed up the research on non-coding RNAs in the transcription regulation of DAPKs in the future.

\section{AUTHOR CONTRIBUTIONS}

QW: Writing-original draft. YL: Conceptualization; Data curation. WZ: Resources; Software. YJ and YL: Writing-review and editing. 


\section{FUNDING}

This research was funded by the National Science Foundation for Young Scientists of China (82003095), the fund of Natural Science Foundation of Fujian Province (2020J01311402), the fund of Fujian Provincial Key Laboratory of Hepatic Drug Research (KFLX2020001), scientific research innovation team construction

\section{REFERENCES}

Agarwal, V., Bell, G. W., Nam, J. W., and Bartel, D. P. (2015). Predicting Effective microRNA Target Sites in Mammalian mRNAs. Elife 4. doi:10.7554/elife.05005

Anaparti, V., Smolik, I., Meng, X., Spicer, V., Mookherjee, N., and El-Gabalawy, H. (2017). Whole Blood microRNA Expression Pattern Differentiates Patients with Rheumatoid Arthritis, Their Seropositive First-Degree Relatives, and Healthy Unrelated Control Subjects. Arthritis Res. Ther. 19 (1), 249. doi:10.1186/s13075-017-1459-x

Ban, E., Jeong, S., Park, M., Kwon, H., Park, J., Song, E. J., et al. (2020). Accelerated Wound Healing in Diabetic Mice by miRNA-497 and its Anti-inflammatory Activity. Biomed. Pharmacother. 121, 109613. doi:10.1016/ j.biopha.2019.109613

Benderska, N., Ivanovska, J., Rau, T. T., Schulze-Luehrmann, J., Mohan, S., Chakilam, S., et al. (2014). DAPK-HSF1 Interaction as a Positive-Feedback Mechanism Stimulating TNF-Induced Apoptosis in Colorectal Cancer Cells. J. Cell Sci. 127 (Pt 24), 5273-5287. doi:10.1242/jcs.157024

Bi, W., Huang, J., Nie, C., Liu, B., He, G., Han, J., et al. (2018). CircRNA circRNA_102171 Promotes Papillary Thyroid Cancer Progression through Modulating CTNNBIP1-dependent Activation of $\beta$-catenin Pathway. J. Exp. Clin. Cancer Res. CR 37 (1), 275. doi:10.1186/s13046-018-0936-7

Botti, G., Marra, L., Malzone, M. G., Anniciello, A., Botti, C., Franco, R., et al. (2017). LncRNA HOTAIR as Prognostic Circulating Marker and Potential Therapeutic Target in Patients with Tumor Diseases. Curr. Drug Targets 18 (1), 27-34. doi:10.2174/1389450117666151209122950

Brock, M. V., Gou, M., Akiyama, Y., Muller, A., Wu, T. T., Montgomery, E., et al. (2003). Prognostic Importance of Promoter Hypermethylation of Multiple Genes in Esophageal Adenocarcinoma. Clin. Cancer Res. 9 (8), 2912-2919.

Brognard, J., Zhang, Y.-W., Puto, L. A., and Hunter, T. (2011). Cancer-Associated Loss-Of-Function Mutations Implicate DAPK3 as a Tumor-Suppressing Kinase. Cancer Res. 71 (8), 3152-3161. doi:10.1158/0008-5472.can-10-3543

Bushati, N., and Cohen, S. M. (2007). microRNA Functions. Annu. Rev. Cell Dev. Biol. 23, 175-205. doi:10.1146/annurev.cellbio.23.090506.123406

Cai, F., Xiao, X., Niu, X., and Zhong, Y. (2017). Association between Promoter Methylation of DAPK Gene and HNSCC: A Meta-Analysis. PLoS One 12 (3), e0173194. doi:10.1371/journal.pone.0173194

Cai, Z., Cao, R., Zhang, K., Xue, Y., Zhang, C., Zhou, Y., et al. (2015). Oncogenic miR-17/20a Forms a Positive Feed-Forward Loop with the P53 Kinase DAPK3 to Promote Tumorigenesis. J. Biol. Chem. 290 (32), 19967-19975. doi:10.1074/ jbc.m115.661504

Cao, M., Zhang, L., Wang, J.-H., Zeng, H., Peng, Y., Zou, J., et al. (2019). Identifying circRNA-Associated-ceRNA Networks in Retinal Neovascularization in Mice. Int. J. Med. Sci. 16 (10), 1356-1365. doi:10.7150/ijms.35149

Cech, T. R., and Steitz, J. A. (2014). The Noncoding RNA Revolution-Trashing Old Rules to Forge New Ones. Cell 157 (1), 77-94. doi:10.1016/j.cell.2014.03.008

Chen, B., Xu, P., Wang, J., and Zhang, C. (2019). The Role of MiRNA in Polycystic Ovary Syndrome (PCOS). Gene 706, 91-96. doi:10.1016/j.gene.2019.04.082

Chen, D., Ma, W., Ke, Z., and Xie, F. (2018b). CircRNA Hsa_circ_100395 Regulates miR-1228/TCF21 Pathway to Inhibit Lung Cancer Progression. Cell Cycle 17 (16), 2080-2090. doi:10.1080/15384101.2018.1515553

Chen, H.-Y., Lee, Y.-R., and Chen, R.-H. (2014). The Functions and Regulations of DAPK in Cancer Metastasis. Apoptosis 19 (2), 364-370. doi:10.1007/s10495013-0923-6

Chen, J., Huang, X., Wang, W., Xie, H., Li, J., Hu, Z., et al. (2018a). LncRNA CDKN2BAS Predicts Poor Prognosis in Patients with Hepatocellular program of Fujian Normal University (IRTL1702), the Special Funds of the Central Government Guiding Local Science and Technology Development (2020L3008), Young Talent Support Program of Women and Children's Hospital, School of Medicine, Xiamen University ((2018)26); Lin Qiaozhi Scientific Research Project of Women and Children's Hospital, School of Medicine, Xiamen University (General Program, FYLQZ2020002).

Carcinoma and Promotes Metastasis via the miR-153-5p/ARHGAP18 Signaling axis. Aging 10 (11), 3371-3381. doi:10.18632/aging.101645

Chen, L.-L. (2016). Linking Long Noncoding RNA Localization and Function. Trends Biochemical Sciences 41 (9), 761-772. doi:10.1016/j.tibs.2016.07.003

Chen, L.-L., and Yang, L. (2015). Regulation of circRNA Biogenesis. RNA Biol. 12 (4), 381-388. doi:10.1080/15476286.2015.1020271

Chen, Q., Liu, T., Bao, Y., Zhao, T., Wang, J., Wang, H., et al. (2020). CircRNA cRAPGEF5 Inhibits the Growth and Metastasis of Renal Cell Carcinoma via the miR-27a-3p/TXNIP Pathway. Cancer Lett. 469, 68-77. doi:10.1016/ j.canlet.2019.10.017

Christoph, F., Kempkensteffen, C., Weikert, S., Köllermann, J., Krause, H., Miller, K., et al. (2006). Methylation of Tumour Suppressor Genes APAF-1 and DAPK1 and In Vitro Effects of Demethylating Agents in Bladder and Kidney Cancer. Br. J. Cancer 95 (12), 1701-1707. doi:10.1038/sj.bjc.6603482

Collins, Y., Dicioccio, R., Keitz, B., Lele, S., and Odunsi, K. (2006). Methylation of Death-Associated Protein Kinase in Ovarian Carcinomas. Int. J. Gynecol. Cancer official J. Int. Gynecol. Cancer Soc. 16 (Suppl. 1), 195-199. doi:10.1111/j.1525-1438.2006.00506.x

Costa, M. C., Cortez-Dias, N., Gabriel, A., de Sousa, J., Fiúza, M., Gallego, J., et al. (2019). circRNA-miRNA Cross-Talk in the Transition from Paroxysmal to Permanent Atrial Fibrillation. Int. J. Cardiol. 290, 134-137. doi:10.1016/ j.ijcard.2019.04.072

Cui, Z., An, X., Li, J., Liu, Q., and Liu, W. (2018). LncRNA MIR22HG Negatively Regulates miR-141-3p to Enhance DAPK1 Expression and Inhibits Endometrial Carcinoma Cells Proliferation. Biomed. Pharmacother. 104, 223-228. doi:10.1016/j.biopha.2018.05.046

Das, T. P., Suman, S., Papu John, A. M. S., Pal, D., Edwards, A., Alatassi, H., et al. (2016). Activation of AKT Negatively Regulates the Pro-apoptotic Function of Death-Associated Protein Kinase 3 (DAPK3) in Prostate Cancer. Cancer Lett. 377 (2), 134-139. doi:10.1016/j.canlet.2016.04.028

Esposito, C. L., Catuogno, S., and de Franciscis, V. (2016). Aptamer-MiRNA Conjugates for Cancer Cell-Targeted Delivery. Methods Mol. Biol. (Clifton, NJ) 1364, 197-208. doi:10.1007/978-1-4939-3112-5_16

Fan, K., Zebisch, A., Horny, K., Schrama, D., and Becker, J. C. (2020). Highly Expressed miR-375 Is Not an Intracellular Oncogene in Merkel Cell Polyomavirus-Associated Merkel Cell Carcinoma. Cancers (Basel) 12 (3). doi:10.3390/cancers12030529

Fang, G., Ye, B.-L., Hu, B.-R., Ruan, X.-J., and Shi, Y.-X. (2018). CircRNA_100290 Promotes Colorectal Cancer Progression through miR-516b-Induced Downregulation of FZD4 Expression and Wnt $\beta$-Catenin Signaling. Biochem. Biophysical Res. Commun. 504 (1), 184-189. doi:10.1016/ j.bbrc.2018.08.152

Feng, L., Li, J., Li, F., Li, H., Bei, S., Zhang, X., et al. (2020). Long Noncoding RNA VCAN-AS1 Contributes to the Progression of Gastric Cancer via Regulating P53 Expression. J. Cell Physiol. 235 (5), 4388-4398. doi:10.1002/jcp.29315

Flatley, J. E., Sargent, A., Kitchener, H. C., Russell, J. M., and Powers, H. J. (2014). Tumour Suppressor Gene Methylation and Cervical Cell Folate Concentration Are Determinants of High-Risk Human Papillomavirus Persistence: a Nested Case Control Study. BMC cancer 14, 803. doi:10.1186/1471-2407-14-803

Fridrichova, I., and Zmetakova, I. (2019). MicroRNAs Contribute to Breast Cancer Invasiveness. Cells 8 (11), 1361. doi:10.3390/cells8111361

Fujita, Y., and Yamashita, T. (2014). Role of DAPK in Neuronal Cell Death. Apoptosis 19 (2), 339-345. doi:10.1007/s10495-013-0917-4

Gandesiri, M., Chakilam, S., Ivanovska, J., Benderska, N., Ocker, M., Di Fazio, P. et al. (2012). DAPK Plays an Important Role in Panobinostat-Induced Autophagy and Commits Cells to Apoptosis under Autophagy Deficient Conditions. Apoptosis 17 (12), 1300-1315. doi:10.1007/s10495-012-0757-7 
Gao, X., Wang, H., Pollok, K. E., Chen, J., and Cohen-Gadol, A. A. (2015). Activation of Death-Associated Protein Kinase in Human Peritumoral Tissue: A Potential Therapeutic Target. J. Clin. Neurosci. 22 (10), 1655-1660. doi:10.1016/j.jocn.2015.03.044

Glažar, P., Papavasileiou, P., and Rajewsky, N. (2014). circBase: a Database for Circular RNAs. RNA 20 (11), 1666-1670.

Guo, Y., Liu, J., Elfenbein, S. J., Ma, Y., Zhong, M., Qiu, C., et al. (2015). Characterization of the Mammalian miRNA Turnover Landscape. Nucleic Acids Res. 43 (4), 2326-2341. doi:10.1093/nar/gkv057

Harder, J., Opitz, O. G., Brabender, J., Olschewski, M., Blum, H. E., Nomoto, S., et al. (2008). Quantitative Promoter Methylation Analysis of Hepatocellular Carcinoma, Cirrhotic and normal Liver. Int. J. Cancer 122 (12), 2800-2804. doi:10.1002/ijc.23433

Harrison, B., Kraus, M., Burch, L., Stevens, C., Craig, A., Gordon-Weeks, P., et al. (2008). DAPK-1 Binding to a Linear Peptide Motif in MAP1B Stimulates Autophagy and Membrane Blebbing. J. Biol. Chem. 283 (15), 9999-10014. doi:10.1074/jbc.m706040200

Hsiao, K.-Y., Sun, H. S., and Tsai, S.-J. (2017). Circular RNA - New Member of Noncoding RNA with Novel Functions. Exp. Biol. Med. (Maywood) 242 (11), 1136-1141. doi:10.1177/1535370217708978

Huang, Y., Chen, L., Guo, L., Hupp, T. R., and Lin, Y. (2014). Evaluating DAPK as a Therapeutic Target. Apoptosis 19 (2), 371-386. doi:10.1007/s10495-0130919-2

Humbert, M., Federzoni, E. A., Britschgi, A., Schläfli, A. M., Valk, P. J. M., Kaufmann, T., et al. (2014). The Tumor Suppressor Gene DAPK2 Is Induced by the Myeloid Transcription Factors PU.1 and C/EBP during Granulocytic Differentiation but Repressed by PML-RAR in APL. J. Leukoc. Biol. 95 (1), 83-93. doi:10.1189/jlb.1112608

Iwakiri, J., Terai, G., and Hamada, M. (2017). Computational Prediction of IncRNA-mRNA Interactionsby Integrating Tissue Specificity in Human Transcriptome. Biol. direct 12 (1), 15. doi:10.1186/s13062-017-0183-4

Jathar, S., Kumar, V., Srivastava, J., and Tripathi, V. (2017). Technological Developments in IncRNA Biology. Adv. Exp. Med. Biol. 1008, 283-323. doi:10.1007/978-981-10-5203-3_10

Jia, Y., Duan, Y., Liu, T., Wang, X., Lv, W., Wang, M., et al. (2019). LncRNA TTNAS1 Promotes Migration, Invasion, and Epithelial Mesenchymal Transition of Lung Adenocarcinoma via Sponging miR-142-5p to Regulate CDK5. Cell Death Dis. 10 (8), 573. doi:10.1038/s41419-019-1811-y

Jing, Z. F., Bi, J. B., Li, Z., Liu, X., Li, J., Zhu, Y., et al. (2019). Inhibition of miR-34a$5 p$ Can rescue Disruption of the p53-DAPK axis to Suppress Progression of clear Cell Renal Cell Carcinoma. Mol. Oncol. 13 (10), 2079-2097. doi:10.1002/ 1878-0261.12545

Kilinc, D., Ozdemir, O., Ozdemir, S., Korgali, E., Koksal, B., Uslu, A., et al. (2012). Alterations in Promoter Methylation Status of Tumor SuppressorHIC1,SFRP2, andDAPK1Genes in Prostate Carcinomas. DNA Cell Biol. 31 (5), 826-832. doi:10.1089/dna.2011.1431

Krajnović, M., Radojković, M., Davidović, R., Dimitrijević, B., and Krtolica, K. (2013). Prognostic Significance of Epigenetic Inactivation of P16, P15, MGMT and DAPK Genes in Follicular Lymphoma. Med. Oncol. (Northwood, Lond. England) 30 (1), 441. doi:10.1007/s12032-012-0441-3

Kuo, J.-C., Wang, W.-J., Yao, C.-C., Wu, P.-R., and Chen, R.-H. (2006). The Tumor Suppressor DAPK Inhibits Cell Motility by Blocking the Integrin-Mediated Polarity Pathway. J. cell Biol. 172 (4), 619-631. doi:10.1083/jcb.200505138

Lai, M.-Z., and Chen, R.-H. (2014). Regulation of Inflammation by DAPK. Apoptosis 19 (2), 357-363. doi:10.1007/s10495-013-0933-4

Lehmann, U., Celikkaya, G., Hasemeier, B., Länger, F., and Kreipe, H. (2002). Promoter Hypermethylation of the Death-Associated Protein Kinase Gene in Breast Cancer Is Associated with the Invasive Lobular Subtype. Cancer Res. 62 (22), 6634-6638.

Li, D., Xu, D., Xu, Y., Chen, L., Li, C., Dai, X., et al. (2017b). MicroRNA-141-3p Targets DAPK1 and Inhibits Apoptosis in Rat Ovarian Granulosa Cells. Cell Biochem Funct 35 (4), 197-201. doi:10.1002/cbf.3248

Li, H., Wang, M., Zhou, H., Lu, S., and Zhang, B. (2020). Long Noncoding RNA EBLN3P Promotes the Progression of Liver Cancer via Alteration of microRNA-144-3p/DOCK4 Signal. Cmar 12, 9339-9349. doi:10.2147/ cmar.s261976
Li, J., Li, Z., Zheng, W., Li, X., Wang, Z., Cui, Y., et al. (2017a). LncRNA-ATB: An Indispensable Cancer-Related Long Noncoding RNA. Cell Prolif. 50 (6), e12381. doi:10.1111/cpr.12381

Li, J.-H., Liu, S., Zhou, H., Qu, L.-H., and Yang, J.-H. (2014). starBase v2.0: Decoding miRNA-ceRNA, miRNA-ncRNA and Protein-RNA Interaction Networks from Large-Scale CLIP-Seq Data. Nucl. Acids Res. 42 (Database issue), D92-D97. doi:10.1093/nar/gkt1248

Li, S., Xiao, F.-Y., Shan, P.-R., Su, L., Chen, D.-L., Ding, J.-Y., et al. (2015). Overexpression of microRNA-133a Inhibits Ischemia-Reperfusion-Induced Cardiomyocyte Apoptosis by Targeting DAPK2. J. Hum. Genet. 60 (11), 709-716. doi:10.1038/jhg.2015.96

Liao, K., Xu, J., Yang, W., You, X., Zhong, Q., and Wang, X. (2018). The Research Progress of LncRNA Involved in the Regulation of Inflammatory Diseases. Mol. Immunol. 101, 182-188. doi:10.1016/j.molimm.2018.05.030

Liao, Y., Li, P., Wang, Y., Chen, H., Ning, S., and Su, D. (2020). Construction of Asthma Related Competing Endogenous RNA Network Revealed Novel Long Non-coding RNAs and Potential New Drugs. Respir. Res. 21 (1), 14. doi:10.1186/s12931-019-1257-x

Lin, X., and Chen, Y. (2018). Identification of Potentially Functional CircRNAmiRNA-mRNA Regulatory Network in Hepatocellular Carcinoma by Integrated Microarray Analysis. Med. Sci. Monit. Basic Res. 24, 70-78. doi:10.12659/msmbr.909737

Lin, Y., Stevens, C., Harrison, B., Pathuri, S., Amin, E., and Hupp, T. R. (2009). The Alternative Splice Variant of DAPK-1, S-DAPK-1, Induces Proteasomeindependent DAPK-1 Destabilization. Mol. Cell Biochem. 328 (1-2), 101-107. doi:10.1007/s11010-009-0079-4

Lin, Y., Stevens, C., and Hupp, T. (2007). Identification of a Dominant Negative Functional Domain on DAPK-1 that Degrades DAPK-1 Protein and Stimulates TNFR-1-Mediated Apoptosis. J. Biol. Chem. 282 (23), 16792-16802. doi:10.1074/jbc.m611559200

Liu, F., Huang, W., Hong, J., Cai, C., Zhang, W., Zhang, J., et al. (2020). Long Noncoding RNA LINC00630 Promotes Radio-resistance by Regulating BEX1 Gene Methylation in Colorectal Cancer Cells. IUBMB Life 72 (7), 1404-1414. doi:10.1002/iub.2263

Liu, G., and Li, B. (2019). Role of miRNA in Transformation from normal Tissue to Colorectal Adenoma and Cancer. J. Cancer Res. Ther. 15 (2), 278-285. doi:10.4103/jcrt.JCRT_135_18

Liu, W.-L., Yang, H.-C., Hsu, C.-S., Wang, C.-C., Wang, T.-S., Kao, J.-H., et al. (2016). Pegylated IFN- $\alpha$ Suppresses Hepatitis C Virus by Promoting the DAPK-mTOR Pathway. Proc. Natl. Acad. Sci. USA. 113 (51), 14799-14804. doi:10.1073/pnas.1618517114

Liu, Z., Yu, Y., Huang, Z., Kong, Y., Hu, X., Xiao, W., et al. (2019). CircRNA-5692 Inhibits the Progression of Hepatocellular Carcinoma by Sponging miR-328-5p to Enhance DAB2IP Expression. Cell Death Dis. 10 (12), 900. doi:10.1038/ s41419-019-2089-9

Lu, Q., Liu, T., Feng, H., Yang, R., Zhao, X., Chen, W., et al. (2019). Circular RNA circSLC8A1 Acts as a Sponge of miR-130b/miR-494 in Suppressing Bladder Cancer Progression via Regulating PTEN. Mol. Cancer 18 (1), 111. doi:10.1186/ s12943-019-1040-0

Luo, T., Zhao, J., Lu, Z., Bi, J., Pang, T., Cui, H., et al. (2018). Characterization of Long Non-coding RNAs and MEF2C-AS1 Identified as a Novel Biomarker in Diffuse Gastric Cancer. Translational Oncol. 11 (5), 1080-1089. doi:10.1016/ j.tranon.2018.06.007

Michlewski, G., and Cáceres, J. F. (2019). Post-transcriptional Control of miRNA Biogenesis. Rna 25 (1), 1-16. doi:10.1261/rna.068692.118

Militello, G., Weirick, T., John, D., Döring, C., Dimmeler, S., and Uchida, S. (2017). Screening and Validation of lncRNAs and circRNAs as miRNA Sponges. Brief Bioinform 18 (5), 780-788. doi:10.1093/bib/bbw053

Pronina, I. V., Loginov, V. I., Burdennyy, A. M., Fridman, M. V., Senchenko, V. N., Kazubskaya, T. P., et al. (2017). DNA Methylation Contributes to Deregulation of 12 Cancer-Associated microRNAs and Breast Cancer Progression. Gene 604, 1-8. doi:10.1016/j.gene.2016.12.018

Raval, A., Tanner, S. M., Byrd, J. C., Angerman, E. B., Perko, J. D., Chen, S.-S., et al. (2007). Downregulation of Death-Associated Protein Kinase 1 (DAPK1) in Chronic Lymphocytic Leukemia. Cell 129 (5), 879-890. doi:10.1016/ j.cell.2007.03.043 
Renganathan, A., and Felley-Bosco, E. (2017). Long Noncoding RNAs in Cancer and Therapeutic Potential. Adv. Exp. Med. Biol. 1008, 199-222. doi:10.1007/ 978-981-10-5203-3_7

Rennier, K., and Ji, J. Y. (2013b). Effect of Shear Stress and Substrate on Endothelial DAPK Expression, Caspase Activity, and Apoptosis. BMC Res. Notes 6, 10. doi:10.1186/1756-0500-6-10

Rennier, K., and Ji, J. Y. (2015). Shear Stress Attenuates Apoptosis Due to TNFa, Oxidative Stress, and Serum Depletion via Death-Associated Protein Kinase (DAPK) Expression. BMC Res. Notes 8, 85. doi:10.1186/ s13104-015-1037-8

Rennier, K., and Ji, J. Y. (2013a). The Role of Death-Associated Protein Kinase (DAPK) in Endothelial Apoptosis under Fluid Shear Stress. Life Sci. 93 (5-6), 194-200. doi:10.1016/j.lfs.2013.06.011

Rizzi, M., Tschan, M. P., Britschgi, C., Britschgi, A., Hügli, B., Grob, T. J., et al. (2007). The Death-Associated Protein Kinase 2 Is Up-Regulated during normal Myeloid Differentiation and Enhances Neutrophil Maturation in Myeloid Leukemic Cells. J. Leukoc. Biol. 81 (6), 1599-1608. doi:10.1189/jlb.0606400

Sanchez-Cespedes, M., Esteller, M., Wu, L., Nawroz-Danish, H., Yoo, G. H., Koch, W. M., et al. (2000). Gene Promoter Hypermethylation in Tumors and Serum of Head and Neck Cancer Patients. Cancer Res. 60 (4), 892-895.

Shiloh, R., Bialik, S., and Kimchi, A. (2014). The DAPK Family: a StructureFunction Analysis. Apoptosis 19 (2), 286-297. doi:10.1007/s10495-013-0924-5

Soghli, N., Yousefi, T., Abolghasemi, M., and Qujeq, D. (2021). NORAD, a Critical Long Non-coding RNA in Human Cancers. Life Sci. 264, 118665. doi:10.1016/ j.lfs. 2020.118665

Steinmann, S., Kunze, P., Hampel, C., Eckstein, M., Bertram Bramsen, J., Muenzner, J. K., et al. (2019). DAPK1 Loss Triggers Tumor Invasion in Colorectal Tumor Cells. Cell Death Dis. 10 (12), 895. doi:10.1038/s41419019-2122-z

Stevens, C., Lin, Y., Harrison, B., Burch, L., Ridgway, R. A., Sansom, O., et al. (2009). Peptide Combinatorial Libraries Identify TSC2 as a Death-Associated Protein Kinase (DAPK) Death Domain-Binding Protein and Reveal a Stimulatory Role for DAPK in mTORC1 Signaling. J. Biol. Chem. 284 (1), 334-344. doi:10.1074/jbc.m805165200

Su, C.-M., Wang, M.-Y., Hong, C.-C., Chen, H.-A., Su, Y.-H., Wu, C.-H., et al. (2016). miR-520h Is Crucial for DAPK2 Regulation and Breast Cancer Progression. Oncogene 35 (9), 1134-1142. doi:10.1038/onc.2015.168

Su, Y., Deng, M.-F., Xiong, W., Xie, A.-J., Guo, J., Liang, Z.-H., et al. (2019). MicroRNA-26a/Death-Associated Protein Kinase 1 Signaling Induces Synucleinopathy and Dopaminergic Neuron Degeneration in Parkinson's Disease. Biol. Psychiatry 85 (9), 769-781. doi:10.1016/j.biopsych.2018.12.008

Sun, Z., Shi, K., Yang, S., Liu, J., Zhou, Q., Wang, G., et al. (2018). Effect of Exosomal miRNA on Cancer Biology and Clinical Applications. Mol. Cancer 17 (1), 147. doi:10.1186/s12943-018-0897-7

Sur, S., Steele, R., Shi, X., and Ray, R. B. (2019). miRNA-29b Inhibits Prostate Tumor Growth and Induces Apoptosis by Increasing Bim Expression. Cells 8 (11), 1455. doi:10.3390/cells8111455

Tian, X., Xu, L., and Wang, P. (2015). MiR-191 Inhibits TNF- $\alpha$ Induced Apoptosis of Ovarian Endometriosis and Endometrioid Carcinoma Cells by Targeting DAPK1. Int. J. Clin. Exp. Pathol. 8 (5), 4933-4942.

Tian, X., Shi, Y., Liu, N., Yan, Y., Li, T., Hua, P., et al. (2016). Upregulation of DAPK Contributes to Homocysteine-Induced Endothelial Apoptosis via the Modulation of Bcl2/Bax and Activation of Caspase 3. Mol. Med. Rep. 14 (5), 4173-4179. doi:10.3892/mmr.2016.5733

Tian, Y., Yan, M., Zheng, J., Li, R., Lin, J., Xu, A., et al. (2019). miR-483-5p Decreases the Radiosensitivity of Nasopharyngeal Carcinoma Cells by Targeting DAPK1. Lab. Invest. 99 (5), 602-611. doi:10.1038/s41374-018$0169-6$

Vastrad, B., Vastrad, C., Godavarthi, A., and Chandrashekar, R. (2017). Molecular Mechanisms Underlying Gliomas and Glioblastoma Pathogenesis Revealed by Bioinformatics Analysis of Microarray Data. Med. Oncol. (Northwood, Lond. England) 34 (11), 182. doi:10.1007/s12032-017-1043-x

Villanova, L., Barbini, C., Piccolo, C., Boe, A., De Maria, R., and Fiori, M. E. (2020). miR-1285-3p Controls Colorectal Cancer Proliferation and Escape from Apoptosis through DAPK2. Int. J. Mol. Sci. 21 (7). doi:10.3390/ijms21072423

Wang, J., Su, Z., Lu, S., Fu, W., Liu, Z., Jiang, X., et al. (2018b). LncRNA HOXA-AS2 and its Molecular Mechanisms in Human Cancer. Clinica Chim. Acta 485, 229-233. doi:10.1016/j.cca.2018.07.004
Wang, P., Li, X., Gao, Y., Guo, Q., Wang, Y., Fang, Y., et al. (2019). LncACTdb 2.0: an Updated Database of Experimentally Supported ceRNA Interactions Curated from Low- and High-Throughput Experiments. Nucleic Acids Res. 47 (D1), D121-d127. doi:10.1093/nar/gky1144

Wang, Y. H., Luo, Z. H., Wang, Z. C., You, M. H., Xie, S. S., Peng, Y. R., et al. (2018a). Effect of Curcumin-Loaded Nanoparticles on Mitochondrial Dysfunctions of Breast Cancer Cells. J. Nanoparticle Res. 20 (10). doi:10.1007/s11051-018-4382-4

Wei, R., Zhang, L., Hu, W., Wu, J., and Zhang, W. (2019). Long Non-coding RNA AK038897 Aggravates Cerebral Ischemia/reperfusion Injury via Acting as a ceRNA for miR-26a-5p to Target DAPK1. Exp. Neurol. 314, 100-110. doi:10.1016/j.expneurol.2019.01.009

Xie, J. Y., Chen, P. C., Zhang, J. L., Gao, Z. S., Neves, H., Zhang, S. D., et al. (2017). The Prognostic Significance of DAPK1 in Bladder Cancer. PLoS One 12 (4), e0175290. doi:10.1371/journal.pone. 0175290

Xing, Z., Park, P. K., Lin, C., and Yang, L. (2015). LncRNA BCAR4 Wires up Signaling Transduction in Breast Cancer. RNA Biol. 12 (7), 681-689. doi:10.1080/15476286.2015.1053687

Xu, X., Wang, X., Geng, C., Nie, X., and Bai, C. (2017). Long-chain Non-coding RNA DAPK1 Targeting miR-182 Regulates Pancreatic Cancer Invasion and Metastasis through ROCK-1/rhoa Signaling Pathway. Int. J. Clin. Exp. Pathol. 10 (9), 9273-9283.

Yan, A., Chen, G., and Nie, J. (2020). DGUOK-AS1 Promotes the Proliferation Cervical Cancer through Regulating miR-653-5p/EMSY. Cancer Biol. Ther. 29, 1-9. doi:10.1080/15384047.2020.1775445

Yan, L.-H., Chen, Z.-N., Li-Li, L., Chen, J., Wei, W.-E., Mo, X.-W., et al. (2016b) miR-135a Promotes Gastric Cancer Progression and Resistance to Oxaliplatin. Oncotarget 7 (43), 70699-70714. doi:10.18632/oncotarget.12208

Yan, L. H., Chen, Z. N., Li, L., Chen, J., Mo, X. W., Qin, Y. Z., et al. (2016a). E2F-1 Promotes DAPK2-Induced Anti-tumor Immunity of Gastric Cancer Cells by Targeting miR-34a. Tumour Biol. 37, 15925-15936. doi:10.1007/s13277-016-5446-7

Ye, M., Li, D., Zhou, F., Guo, Q., and Xia, B. (2012). Epigenetic Regulation of Death-Associated Protein Kinase Expression in Primary Gastric Cancers from Chinese Patients. Eur. J. Cancer Prev. official J. Eur. Cancer Prev. Organisation (Ecp) 21 (3), 241-246. doi:10.1097/cej.0b013e32834c9caa

Ye, S. S., Liu, X. J., Mao, B. Y., Yang, L., and Qiu, G. (2016). Value of DAPK Gene Methylation Patterns in the Diagnosis of Leukemia. Zhongguo shi yan xue ye xue za zhi 24 (3), 687-692. doi:10.7534/j.issn.1009-2137.2016.03.009

Ye, Y., Shen, A., and Liu, A. (2019). Long Non-coding RNA H19 and Cancer: A Competing Endogenous RNA. Bull. $d u$ Cancer 106 (12), 1152-1159. doi:10.1016/j.bulcan.2019.08.011

Yin, S., Dou, J., Yang, G., and Chen, F. (2019). Long Non-coding RNA XIST Expression as a Prognostic Factor in Human Cancers: A Meta-Analysis. Int. J. Biol. Markers 34 (4), 327-333. doi:10.1177/1724600819873010

Yu, L., and Liu, Y. (2019). circRNA_0016624 Could Sponge miR-98 to Regulate BMP2 Expression in Postmenopausal Osteoporosis. Biochem. Biophysical Res. Commun. 516 (2), 546-550. doi:10.1016/j.bbrc.2019.06.087

Yuan, S., Liang, J., Zhang, M., Zhu, J., Pan, R., Li, H., et al. (2019). CircRNA_005647 Inhibits Expressions of Fibrosis-Related Genes in Mouse Cardiac Fibroblasts via Sponging miR-27b-3p. Nan Fang Yi Ke Da Xue Xue Bao 39 (11), 1312-1319. doi:10.12122/j.issn.1673-4254.2019.11.08

Yung, C., MacDonald, T. M., Walker, S. P., Cannon, P., Harper, A., Pritchard, N., et al. (2019). Death Associated Protein Kinase 1 (DAPK-1) Is Increased in Preeclampsia. Placenta 88, 1-7. doi:10.1016/j.placenta.2019.09.010

Zhai, C.-L., Tang, G.-M., Qian, G., Hu, H.-L., Wang, S.-J., Yin, D., et al. (2019). MicroRNA-98 Attenuates Cardiac Ischemia-Reperfusion Injury through Inhibiting DAPK1 Expression. IUBMB Life 71 (2), 166-176. doi:10.1002/iub.1879

Zhang, F., Zhang, R., Zhang, X., Wu, Y., Li, X., Zhang, S., et al. (2018b). Comprehensive Analysis of circRNA Expression Pattern and circRNAmiRNA-mRNA Network in the Pathogenesis of Atherosclerosis in Rabbits. Aging 10 (9), 2266-2283. doi:10.18632/aging.101541

Zhang, H.-d., Jiang, L.-h., Sun, D.-w., Hou, J.-c., and Ji, Z.-l. (2018a). CircRNA: a Novel Type of Biomarker for Cancer. Breast Cancer 25 (1), 1-7. doi:10.1007/ s12282-017-0793-9

Zhang, H., Deng, T., Ge, S., Liu, Y., Bai, M., Zhu, K., et al. (2019b). Exosome circRNA Secreted from Adipocytes Promotes the Growth of Hepatocellular Carcinoma by Targeting Deubiquitination-Related USP7. Oncogene 38 (15), 2844-2859. doi:10.1038/s41388-018-0619-z 
Zhang, J., Liu, L., Sun, Y., Xiang, J., Zhou, D., Wang, L., et al. (2016). MicroRNA-520g Promotes Epithelial Ovarian Cancer Progression and Chemoresistance via DAPK2 Repression. Oncotarget 7 (18), 26516-26534. doi:10.18632/oncotarget.8530

Zhang, L., Liao, Y., and Tang, L. (2019a). MicroRNA-34 Family: a Potential Tumor Suppressor and Therapeutic Candidate in Cancer. J. Exp. Clin. Cancer Res. CR 38 (1), 53. doi:10.1186/s13046-019-1059-5

Zhang, M., Shi, Y., Zhang, Y., Wang, Y., Alotaibi, F., Qiu, L., et al. (2020). miRNA5119 Regulates Immune Checkpoints in Dendritic Cells to Enhance Breast Cancer Immunotherapy. Cancer Immunol. Immunothercii 69 (6), 951-967. doi:10.1007/s00262-020-02507-w

Zhao, T., Wang, D., Hu, Y., Zhang, N., Zang, T., and Wang, Y. (2019). Identifying Alzheimer's Disease-Related miRNA Based on Semi-clustering. Cgt 19 (4), 216-223. doi:10.2174/1566523219666190924113737

Zhen, Q., Gao, L. N., Wang, R. F., Chu, W. W., Zhang, Y. X., Zhao, X. J., et al. (2018). LncRNA DANCR Promotes Lung Cancer by Sequestering miR-216a. Cancer Control. J. Moffitt Cancer Cent. 25 (1), 1073274818769849. doi:10.1177/ 1073274818769849

Zhen, Z., Ren, S., Ji, H., Ding, X., Zou, P., and Lu, J. (2019). The lncRNA DAPK-IT1 Regulates Cholesterol Metabolism and Inflammatory Response in Macrophages and Promotes Atherogenesis. Biochem. Biophysical Res. Commun. 516 (4), 1234-1241. doi:10.1016/j.bbrc.2019.06.113

Zheng, R., Liang, J., Lu, J., Li, S., Zhang, G., Wang, X., et al. (2019). Genome-wide Long Non-coding RNAs Identified a Panel of Novel Plasma Biomarkers for Gastric Cancer Diagnosis. Gastric Cancer 22 (4), 731-741. doi:10.1007/s10120-018-00915-7

Zhou, T., Zhong, Y., Hu, Y., Sun, C., Wang, Y., and Wang, G. (2018). PM2.5 Downregulates miR-194-3p and Accelerates Apoptosis in Cigarette-Inflamed Bronchial Epithelium by Targeting Death-Associated Protein Kinase 1. Copd 13, 2339-2349. doi:10.2147/copd.s168629

Zhou, X., Zhang, W., Jin, M., Chen, J., Xu, W., and Kong, X. (2017). lncRNA MIAT Functions as a Competing Endogenous RNA to Upregulate DAPK2 by Sponging miR-22-3p in Diabetic Cardiomyopathy. Cell Death Dis. 8 (7), e2929. doi:10.1038/cddis.2017.321

Zhou, Y., Wang, M., Wu, J., Jie, Z., Chang, S., and Shuang, T. (2015). The Clinicopathological Significance of miR-1307 in Chemotherapy Resistant Epithelial Ovarian Cancer. J. ovarian Res. 8, 23. doi:10.1186/s13048-015-0143-5
Zhu, H., Zhai, B., He, C., Li, Z., Gao, H., Niu, Z., et al. (2020a). LncRNA TTN-AS1 Promotes the Progression of Cholangiocarcinoma via the miR-320a/ neuropilin-1 axis. Cell Death Dis. 11 (8), 637. doi:10.1038/s41419-02002896-x

Zhu, H., Zhao, S., Jiao, R., Wang, H., Tang, R., Wu, X., et al. (2020b). Prognostic and Clinicopathological Significance of SNHG20 in Human Cancers: a MetaAnalysis. Cancer Cell Int 20, 304. doi:10.1186/s12935-020-01403-8

Zhu, L., Ren, T., Zhu, Z., Cheng, M., Mou, Q., Mu, M., et al. (2018). Thymosin- $\beta 4$ Mediates Hepatic Stellate Cell Activation by Interfering with CircRNA0067835/miR-155/FoxO3 Signaling Pathway. Cell Physiol Biochem. 51 (3), 1389-1398. doi:10.1159/000495556

Zhu, Y., Li, S., Wang, Q., Chen, L., Wu, K., Huang, Y., et al. (2017). Quantitative and Correlation Analysis of the DNA Methylation and Expression of DAPK in Breast Cancer. PeerJ 5, e3084. doi:10.7717/peerj.3084

Zou, Y., Sun, Z., and Sun, S. (2020). LncRNA HCG18 Contributes to the Progression of Hepatocellular Carcinoma via miR-214-3p/CENPM axis. J. Biochem. 168 (5), 535-546. doi:10.1093/jb/mvaa073

Conflict of Interest: The authors declare that the research was conducted in the absence of any commercial or financial relationships that could be construed as a potential conflict of interest.

Publisher's Note: All claims expressed in this article are solely those of the authors and do not necessarily represent those of their affiliated organizations, or those of the publisher, the editors and the reviewers. Any product that may be evaluated in this article, or claim that may be made by its manufacturer, is not guaranteed or endorsed by the publisher.

Copyright (c) 2021 Wang, Lin, Zhong, Jiang and Lin. This is an open-access article distributed under the terms of the Creative Commons Attribution License (CC BY). The use, distribution or reproduction in other forums is permitted, provided the original author(s) and the copyright owner(s) are credited and that the original publication in this journal is cited, in accordance with accepted academic practice. No use, distribution or reproduction is permitted which does not comply with these terms. 